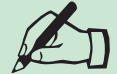

\section{Das Curriculum - ein Politikum}

Eine vorläufige Replik auf den Artikel von M. Geiser [1]

Sehr geehrter, lieber Herr Professor Geiser Vorerst möchte ich Ihnen meine Bewunderung ausdrücken, wie ein seit Jahren emeritierter orthopädischer Chirurg immer wieder bereit ist, sich aktuellen, ja brisanten Themen zu stellen. Wir alle wissen, dass Max Geisser kein einfacher Diskussionspartner ist, aber nur tote Fische schwimmen immer mit dem Strom. Als Vorsitzender der Kommission Fachstudium der Medizinischen Fakultät Bern fühle ich mich durch Ihre Zeilen nicht angegriffen, aber angesprochen. Diese Kommission hat die Aufgabe, das 4.-6. Studienjahr («Klinik») zu planen, zu implementieren und $\mathrm{zu}$ evaluieren. Das 1.-3. Studienjahr wird durch die Kommission Grundstudium (Vorsitz: Prof. U. Brodbeck) geführt. Die beiden Kommissionen unterstehen der Curriculumskommission (Vorsitz: Vizedekan Lehre, Prof. V. Im Hof), welche die strategischen Entscheide fällt. Die Tatsache, dass die medizinische Fakultät Bern mich als Hausarzt zum Vorsitzenden der Kommission Fachstudium gewählt hat, zeigt, dass sie es mit der praktischen Medizin ernst nimmt.

Ich weiss, dass Sie sehr belesen sind und stelle deshalb PANTA REI, alles fliesst, an den Anfang meiner kurzen Replik. Wenn ein Studium in der heutigen Zeit der teilweise rasanten Fortschritte und Entwicklungen in Selbstgefälligkeit erstarrt, werden wir damit den Herausforderungen des Fortschritts nicht gerecht. Es gibt in der Schweiz medizinische Fakultäten, die eher konservativ sind, es gibt Fakultäten, die eher progressiv sind und Bern bewegt sich etwa in der Mitte, aber es bewegt sich! Ein gesunder Mix aus Bewährtem (dazu gehören auch die Vorlesungen, auf die wir nie ganz werden verzichten können) und Neuem (problemorientierter Unterricht, mehr psychosoziale Inhalte, Hausarztmedizin, Ethik, Gesundheitsökonomie usw.) dürfte die angestrebten Studienziele am ehesten in erreichbare Nähe rücken.

So ist auch die gemäss Medien "vernichtende Kritik" der internationalen AkkreditierungsKommission auf verschiedenes Terrain gefallen: Zürich lehnt die Kompetenz der Akkreditierungs-Kommission eher ab, die andern medizinischen Fakultäten versuchen, aus den Erkenntnissen möglichst konstruktive, die lokalen Gegebenheiten berücksichtigende Konsequenzen zu ziehen, was durchaus möglich ist. Es sei an dieser Stelle bewusst nicht auf alle Ihre Kritikpunkte eingegangen, teilweise teile ich Ihre Meinung, teilweise sind Ihre Feststellungen für unsere
Kommission eine Herausforderung.

Auf einen - nichtpolitischen - Punkt Ihres Artikels möchte ich hier bereits eingehen: Ihre Verurteilung des problemorientierten Lernens. Wir sind überzeugt, dass das problemorientierte Lernen - ausgehend von irgendeinem Problem, das der Patient vorbringt, oder das wir feststellen - ein sehr tauglicher Weg ist, die Studierenden anzuleiten, das Problem erst mal zu erkennen, zu werten, ihm auf den Grund zu gehen, $\mathrm{Zu}-$ sammenhänge mit weiteren Problemen aufzudecken und schliesslich Lösungsmöglichkeiten (diagnostisch, therapeutisch) aufzuzeigen. In Bern haben die Studierenden bereits im ersten Semester eine Diskussionskultur und eine Fähigkeit, Ressourcen und verschiedenste Lehrmittel zu nutzen (Vertiefungskompetenz), von der wir früher auf Stufe Staatsexamen nur träumen konnten. Ich will das Rad nicht neu erfinden und verweise auf den kürzlich erschienenen Artikel von Prof. Paul Imbach, Lehrdekan in Basel (NZZ vom 30.10.00, S. 13). Wir werden Sie gerne zu einer Diskussion einladen, in der Meinung, dass kritische, erfahrene Meinungen sehr dazu beitragen können, Neuerungen optimal zu gestalten. Sie werden von uns hören!

Benedikt Horn, Interlaken

1 Geiser M. Das Curriculum - ein Politikum. Schweiz Ärztezeitung 2000;81:2472-5.

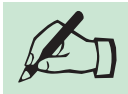

\section{Kontrahierungszwang für Ärzte über 65}

Ich beziehe mich auf einen Artikel aus der Feder von Otto Piller [1].

Wir haben vor vielen Jahren einmal gegeneinander Fussball gespielt, er beim FC Nationalrat und ich beim FC Stadtrat Bern. Ich war damals in der Verteidigung und habe diesen Posten leider jetzt wieder aufnehmen müssen.

Ich führe eine Praxis als Kinder- und Jugendarzt im Zentrum von Bern. Ich lese in seinem Artikel, dass mit zunehmendem Alter die "Wahrscheinlichkeit zunimmt, dass ein Leistungserbringer nicht mehr mit der medizinischen Entwicklung Schritt halten kann und damit die Qualität der Leistungserbringung gefährdet ist». Ich finde diese Aussage als sehr starken persönlichen Affront, da ich mich während meiner ganzen ärztlichen Tätigkeit immer weitergebildet habe und sehr viel Zeit, auch jetzt noch, in meine Weiterbildung stecke! Die Idee, dass bei zunehmendem Alter die Qualität der ärztlichen Leistung gefährdet ist, könnte vielleicht bei gewissen chirurgisch tätigen Ärzten zutreffen.

Ich weiss, dass er und Frau Bundesrätin Dreifuss mit allen Mitteln versuchen, die Kosten des Gesundheitssystems einzudämmen. Ich und ein grosser Teil des Volkes sind diesbezüglich mit ihm sicher einverstanden. Auf der einen Seite will er den Kontrahierungszwang für die über 65jährigen Ärzte aufheben (ohne Kontrahierungszwang kann sicher jeder Arzt seine Praxis schliessen), und auf der anderen Seite will er die jungen Mediziner drei Jahre lang von der Praxiseröffnung fernhalten.

Bei diesen Überlegungen ist allerdings die Logik auf der Strecke geblieben: Auf der einen Seite will er uns älteren Ärzten das Handwerk legen und auf der anderen Seite die jungen, offenbar bestens ausgebildeten Mediziner, die mit der medizinischen Entwicklung Schritt halten, nicht in der Praxis zulassen! Wo liegt da die Logik? In einem Falle will er die älteren Herren, die nicht mehr so ganz richtig im Kopf seien, ausschalten, aber den jungen Medizinern, die uns Älteren offenbar medizinisch haushoch überlegen sind, erlaubt man 3 Jahre lang nicht, nach dem Ende ihrer Ausbildung, eine Praxis zu eröffnen.

Ich habe zwei Kinder, Sohn und Tochter, beides Mediziner, die also jung und geistig noch hochstehend frisch sind, die seine Logik absolut auch nicht verstehen können. Was würde übrigens eingespart, wenn Ärzte über 65 nicht mehr praktizieren könnten? Ich glaube, dass viele ältere Ärzte, wie ich, aufgrund ihrer sehr grossen Erfahrung billiger arbeiten als manch junger Arzt. Aber auch dies müsste mit Zahlen belegt werden können, bevor man zur Streichung des Kontrahierungszwang für Ärzte über 65 schreitet.

Nebenbei möchte ich noch erwähnen, dass er sich bei Herrn Nationalrat Cavalli über mich erkundigen kann: dieser war vor vielen Jahren im Kinderspital in Bern mein Student. Ich habe auch heute noch mit ihm Kontakt. Er kennt mich sehr gut und weiss, dass ich geistig und körperlich noch in bester Form bin.

Um mit Wolf Biermanns Spruch zu schliessen: Auch ein alter Baum trägt noch neue Früchte!

$$
\text { Dr. med. A. Weil, Bern }
$$

1 Piller 0. Das KVG gemeinsam optimieren. Schweiz Ärztezeitung 2000;81(44):2469-71. 


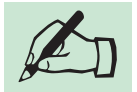

\section{Whiplash/Schleudertrauma - vom Unfall "Schleudertrauma» zur chronischen Krankheit}

Wenn ein Kollege unter diesem Titel einen Artikel schreibt [1], so ist ihm heutzutage die Aufmerksamkeit zahlreicher Leser sicher. Wenn er dann noch die Untertitel «Differentialdiagnose, Co-Morbiditäten, Frühabklärung, Frühtherapie» hinzufügt, so geht der Leser davon aus, dass der Autor langjährige Ausbildung, Erfahrung und breitestes Fachwissen hat, wenn er es wagt, sich so umfassend zu einem derart komplexen Thema zu äussern. Leider war mir der Autor ausser durch eine Publikation im Jahre 1999, die er im oben erwähnten Artikel selber zitiert, und deren Ergebnisse er darin ausführlich wiederholt, bisher nicht bekannt.

Aufgrund seiner Ausführungen muss ich annehmen, dass er eine vorwiegend psychiatrische Ausbildung hat. (Das elektronische Telefonbuch gab leider nur "Dr. med. FMH" preis.) Zumindest wirkten auf mich als Neurologe mit bloss einjähriger Assistentenzeit in Psychiatrie und stetigem Alltagsinteresse an psychischen Problemen meiner Patienten seine Äusserungen bezüglich psychiatrischer Aspekte des "Schleudertraumaproblems" einleuchtend, logisch und kompetent. Es würde mich sehr interessieren, ob die Fachpsychiater, welche sich seit Jahren mit dem Schleudertrauma befassen, all seine Ansichten teilen. Ausser Sigmund Freud zitiert er leider nur wenige Autoren zu deren Untermauerung.

Dass der Artikel von Herrn Kollegen Kissel den Lesern weder zur Differentialdiagnose, noch zu den Co-Morbiditäten umfassend und kompetent Auskunft gibt, zumindest nicht hinsichtlich somatischer Aspekte, und dass der Autor "Neue therapeutische Optionen im Schleudertraumabereich" anbietet, welche sich bei näherem Hinsehen teils als "alter Wein in neuen Schläuchen" und teils als höchst fragwürdig und irgendwo abgeschrieben erweisen, zeigt klar, dass er sich, in zwar durchaus glaubhaft guter Absicht, eindeutig zuviel vorgenommen hat.

Dadurch droht ein meines Erachtens äusserst bemerkenswerter und relevanter Vorschlag des Autors ebensowenig ernst genommen $\mathrm{zu}$ werden, oder ungenügend Beachtung zu finden, nämlich das Postulat der Frühabklärung. Anhand seines eigenen gutachterlichen Patientenkollektivs kommt er zum Schluss, dass bei Frühabklärung zumeist bloss eine halbe, statt eine ganze IVRente resultiert hätte. Auch wenn bei dieser Äusserung ein gewisser Zweckoptimismus zur Erhaltung der MEDAS Luzern nicht zu übersehen ist, so kann ich mich seinem Vorschlag nur anschliessen, dass Patienten mit
Verdacht auf ein erlittenes "Schleudertrauma" der HWS schon sehr früh nach dem Ereignis nicht nur somatisch, sondern auch psychiatrisch exploriert und nötigenfalls behandelt werden sollten, und dass sie zu Dokumentationszwecken auch kompetent und adäquat neuropsychologisch getestet werden sollten, allerdings, ohne allenfalls gefundene kognitive Störungen gleich auf eine leichte traumatische Hirnverletzung zurückzuführen, wie dies in der von ihm allzu einseitig ausgewählten und zitierten Literatur der Fall ist, denn dadurch werden die Patienten erfahrungsgemäss unnötigerweise verängstigt! Ergänzend möchte ich aus somatischer Sicht noch postulieren, dass bei Patienten mit vorgefundenen kognitiven Störungen unverzüglich auch eine mechanische und biomechanische Beurteilung des Unfallereignisses durchgeführt wird, um sich auch ein bestmögliches Bild von der Schwere des Ereignisses selbst machen zu können.

Damit wäre der Ball bei den Unfallversicherern: Sie nämlich hätten es in der Hand, bei jeder eingehenden "Schleudertrauma"-Unfallmeldung eine psychiatrische Exploration und eine neuropsychologische Untersuchung 3 Monate nach dem Ereignis zur Bedingung zu machen, falls bis dann die Beschwerden nicht wieder weitgehend abgeklungen sind!

Prof. Dr. med. O. Meienberg, Binningen

1 Kissel W. Whiplash/Schleudertrauma - vom Unfall «Schleudertrauma» zur chronischen Krankheit. Differentialdiagnose, Co-Morbiditäten, Frühabklärung, Frühtherapie. Schweiz Ärztezeitung 2000;81(49):2803-9.

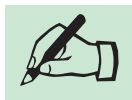

\section{Ungeheuerlichkeiten}

Mit grösster Bestürzung habe ich den Artikel der Kollegen Seebach und Stüssi [1] aufgenommen.

Mit kühler Sprache wird ungeheures Tun an den Leser gebracht, der wortgewandt in ein Denken eingeführt wird, das einem simplen Welt- und Menschenverständnis entspricht. An keiner Stelle wird auch nur im Ansatz ein Zweifel geäussert, ob der Mensch und seine Mitwesen doch noch etwas anderes sein könnten als funktionierende Maschinen. Wörter wie Seele oder Geist sind wohl nicht mehr existent, weil sie nicht existent sein dürfen. Denn man müsste ja allenfalls sich damit auseinandersetzen. Also lieber gar nicht ins Denken einbeziehen. Dadurch wird ein Teil der Bevölkerung fundamental desavouiert, nämlich die Menschen, die noch spirituelle Aspekte ins Leben einfliessen lassen. Ein echter Wissenschaftler wird das Unbekannte (in diesem Beispiel Seele und Geist) erst erforschen wollen, bevor er ausschliesst, dass es existiert. Aber eben ... Das Ganze ist absurd. Frankensteins Medi- zinersöhne haben ein eigenartiges Demokratieverständnis: (teil-)informieren und fragen dann, wenn schon alles eine Eigendynamik bekommen hat. Unter dem Deckmantel "Therapie» wird alles verkauft, was irgendwie aus Eigeninteressen gemacht werden soll. Bis zur Marktreife darf der Staat die Verantwortung übernehmen und bezahlen. Kommt typischerweise dazu, dass unter gesellschaftlichen Gesichtspunkten faktisch nur die epidemiologischen Probleme Bedeutung haben. Ist es so einfach?

Pervers wird es, wenn ein Handlungsauftrag geschustert wird, um sich nicht «dem Vorwurf der unterlassenen Hilfeleistung auszusetzen». Kommentar notwendig? Immerhin werden tierethische Aspekte einbezogen, schliesslich muss man ja.

Um die in der Bundesverfassung festgelegte "Würde der Kreatur» und den Leitsatz der Ärztinnen und Ärzte, «in erster Linie nicht zu schaden", nicht zu missachten, ist es nur konsequent, auf die Xenotransplantation von Organen zu verzichten und die damit zusammenhängende Forschung zu stoppen! Ich bin mir bewusst, mit harten Worten auf den Artikel geantwortet zu haben, aber alles andere wäre nur Augenwischerei.

Dr. med. Markus Scheuring, Bonstetten

PS: von der Vereinigung "Ärztinnen und Ärzte für Tierschutz in der Medizin", Postfach 66, 8053 Zürich, wurde zur Xenotransplantation die Infobroschüre "SchweineMensch oder Menschenschwein" publiziert. Gratis unter aerztinnen.aerzte@bluewin.ch bestellbar.

1 Seebach JD, Stüssi G. Xenotransplantationsforschung - Frankensteins Erbe? Schweiz Ärztezeitung 2000;81(50):2853-8.

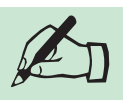

\section{Trotzdem nicht die wahre Lösung}

Zugegeben, die Geschichte von Charlotte Hurni [1] ist amüsant geschrieben, die Lösung eigentlich originell. Doch als Verantwortliche von Betaferon lief mir ein kalter Schauer den Rücken hinunter. Betaferon in Ameisensäure aufgelöst und dann injiziert. Eine bessere Notlösung wäre eine sterile, physiologische Kochsalzlösung, verdünnt mit sterilem Wasser gewesen, eine Apotheke sollte dies herstellen können.

Dr. Martina Sintzel, Schering (Schweiz) AG

1 Hurni C. Die Lösung - Wer mit Betaferon reist, kann etwas erleben ...

Schweiz Ärztezeitung 2000;81(43):2451. 